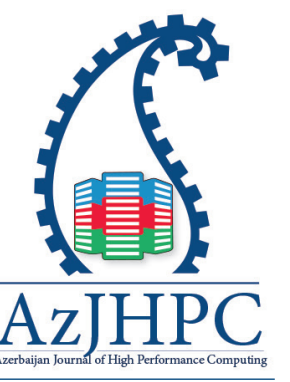

${ }^{*}$ Correspondence: Siddhartha Roy, Faculty of Computer Science, The Heritage College, Calcutta University, Calcutta, India, siddhartha.roy@thc.edu.in

\title{
Automatics Number Plate Recognition Using Convolution Neural Network
}

\author{
Siddhartha Roy \\ Faculty of Computer Science, The Heritage College, Calcutta University, \\ Calcutta, India, siddhartha.roy@thc.edu.in
}

\section{Abstract}

In the last few years, Automatic Number Plate Recognition (ANPR) systems have become widely used for security, safety, and also commercial aspects such as parking control access, and legal steps for the red light violation, highway speed detection, and stolen vehicle detection. The license plate of any vehicle contains a number of numeric characters recognized by the computer. Each country in the world has specific characteristics of the license plate. Due to rapid development in the information system field, the previous manual license plate number writing process in the database is replaced by special intelligent device in a real-time environment. Several approaches and techniques are exploited to achieve better systems accuracy and real-time execution. It is a process of recognizing number plates using Optical Character Recognition (OCR) on images. This paper proposes a deep learning-based approach to detect and identify the Indian number plate automatically. It is based on new computer vision algorithms of both number plate detection and character segmentation. The training needs several images to obtain greater accuracy. Initially, we have developed a training set database by training different segmented characters. Several tests were done by varying the Epoch value to observe the change of accuracy. The accuracy is more than $95 \%$ that presents an acceptable value compared to related works, which is quite satisfactory and recognizes the blurred number plate.

Keyword: ANPR, OCR, CNN, Image Segmentation, Image Recognition, Blurred Image.

\section{Introduction}

At present, the use of vehicles is increasing day by day throughout the country. These vehicles have a unique vehicle identification number, which is the license that refers to a legal license to participate in the public movement. Each vehicle in the world must have its unique number plate installed on its back side and the front side of the body. Identify this number system helps with safety, highway speed detection, red light violation, stolen vehicle detection, car parking system. etc. In the traffic management 
system framework, tracking or surveillance of vehicles on the street has high significance. Vehicles are identified by capturing their number plate and retrieving the data from the database's record. Automobiles can be recognized by number plates, which contain a unique combination of alphabets and numbers. It is challenging for humans to manually recognize and maintain all the parked or passing car number plates. An automatic number plate recognition using programmable software is essential to avoid this tedious work (Panahi, R.and Gholampour, I.,2017). The process of recognizing vehicle license numbers becomes very cost-effective and fast using this automated system (Patel C., Shaw, D., and Patel, A., 2013). The picture can be captured using CCTV street camera or by versatile/handheld cameras used by cops. In order to obtain the license plate identification technique, it requires three steps. The first step is to find the panel of digital images, segmentation the characters from the panel's pictures, and the visual character Recognition (Conci, A., Carvalho, J. E. R. and Rauber, T. W.,2009). The most significant step is to determine the exact location of the number plate in the captured image. Structural analysis and color analysis are used to determine the number plate's location (Abolghasemi, V. and Ahmadyfard, A., 2009). In the License panel area, unnecessary spots are removed by parsing the connected component. The importance of ANPR has increased in recent years, with many applications, including Car vehicles' prediction accuracy and speed. Recent advancement in deep learning has improved its ability to solve complex visual recognition tasks (Jia, Y., Shelhamer, E., Donahue, J., et al., 2014; Krizhevsky, A., Sutskever, I., and Hinton, G. $E ., 2012)$. In this paper, we propose using deep learning techniques to recognize the Indian number plate (which can be further extended to any number plate in the word) and calculate prediction accuracy. We segmented the chartered using OpenCV and created our recognition model by training manually collected car number plate dataset using Convolution Neural Network. The algorithm has been tested over 500 images, which are of different colors and quality. Our preliminary work's objective is to achieve high accuracy, and our system can detect even blurred number plates. After the introductory part in section 1, a brief report on the related work is discussed in Section 2. In section 3, we mention the CNN model evaluation and performance measurement techniques. Section 4 highlights simulation results and its interpretation. The limitation is discussed in Section 5. Finally, the paper is concluded in Section 6, mentioning the future scope of this research work.

\section{Literature Survey}

Several researchers performed various research activities to improve system performance to recognize the car number plate. K. Kaur and V. K. Banga (Kaur, Er. K., and Banga, V. K. 2013) in their paper discussed Optical Character Recognition (OCR) technique for vehicle number identification. Initially, a clear image is obtained without distortion. Then crop the number plate from the captured image, which is used as an input for the character recognition. Finally, character recognition is performed through 
OCR based on template matching. Zhigang Zhang and Cong Wang (Zhang Z. and Wang, C., 2012), in their paper, proposed a new system which is based on a weighted factual technique to detect number plate. In their paper, the authors changed the 24bit shading picture into a grey image. Feng Wang et al. (Wang, F., Man, L., Wang, B., et al., 2000). discussed a four-stage strategy using the fuzzy-based algorithm. In the initial step, the noisy disturbance is removed from the number plate. To find the rectangle area, Edge identification is in the second step. In the third step, resize the picture, histogram, and other data and remove invalid rectangle zones. Geometric correction is used to acquire a tag competitor area in the final stage. Ankush Roy et al. (Roy, A. and Ghoshal, DP,2011) proposed an improved segmentation algorithm. Median filtering, adaptive thresholding, component labeling, region growth, segmentation, and normalization are used to remove noise. The authors used Otsu's technique for picture binarization in the versatile thresholding process. Savitha. K.M, Sadashiva, V.( Savitha, K.M, and Sadashiva, V.C, 2015) proposes a framework for detecting number plate and an artificial Neural Network approach to recognize the characters. This framework has the capacity of distinguishing number plate for varied conditions like typical text dimension, diverse textual style, written by hand characters, distinctive foundation shading for all Indian number plates. The proposed strategy is adaptable and not fragile to picture varieties. G. Abo Samra and F. Khalefah ( Samra, G. A, and Khalefah, $F ., 2014)$ discuss in their paper the threshold edge technique to defeat the dynamic changes of enlightenment conditions while changing over the picture into Connected component analysis technique (CCAT) was utilized to identify the obscure picture. Bo Li, Bin Tian, Ye Li, and Ding Wen in their paper (Li, B., Tian, B. e Li, Y., et al., 2013 ), showing an innovative computation for the throughout the day traffic surveillance condition. Chitode J.S (Kate, R., and Chitode J.S.,2012) suggested that ANPR is an ongoing installed framework that recognizes the characters straightforwardly, which depended on a mix of morphological operation with zone criteria tests for the number plate. A special algorithm is used to divide the OCR engine called a tesseract, which is used to extract ASCII from the license number plate. OpenCV is used to implement the entire system. Raghunandan et al. (Raghunandan, K.S., Shivakumara, P., Kumar, G.H., et al.,2016). Sharpness features for image type classification based on textual information. The method works based on the high quality of captured texts. Xu et al.( Xu, J., Shivakumara, P., Lu, T., et al., 2014) discussed caption and scene text classification in the video, using gradient clustering. However, their proposed method does not work on the scene text. Shivakumara et al. (Shivakumara, P., Kumar, N.V., Guru, D.S., et al., 2014) in their paper shown separation of graphics and scene texts in video frames. In their paper, caption texts have good quality, while scene tests do not. Yang et al.( Yang, Y., Li, D., Duan, Z., 2018) proposed a kernel-based extreme learning machine with deep convolutional features on Chinese vehicle license plate recognition. Contour properties and a deep learning model are proposed in the paper written by Abedin et al.( Abedin, M.Z., Nath, A.C., Dhar, P., et al., 2017). Their model 
segments character accurately for high-quality images but failed to segment for poor quality. Bulan et al. (Bulan, O., Kozitsky, V., Ramesh, P., et al.2017) proposed segmentation and annotation free license plate using hidden Markov model (HMM) for character segmentation and recognition, but it is not clear that how the works well for poor quality images and noises. Selmi et al.( Selmi, Z., Halima, M.B., Alimi, A.M., 2017) proposed a CNN-based model for automatic license plate detection and recognition, which performs many pre-processing steps. This model follows the conventional way of character segmentation and then recognition. This idea is suitable for highquality images and particular types. None of the methods addresses the issues of blur and poor quality. In our work, we have tried to overcome the above-challenges by classifying different types of license plate images using CNN. Detailed work has been discussed in the subsequent sections.

\section{Present Work}

Our entire tasks are mainly divided into two basic steps first step the segmentation of the number plate and recognition of the number plate. Segmentation of number plate is performed using OpenCV, whereas recognition of number plate is performed using CNN. The following steps (Fig 1) are used for our preliminary work

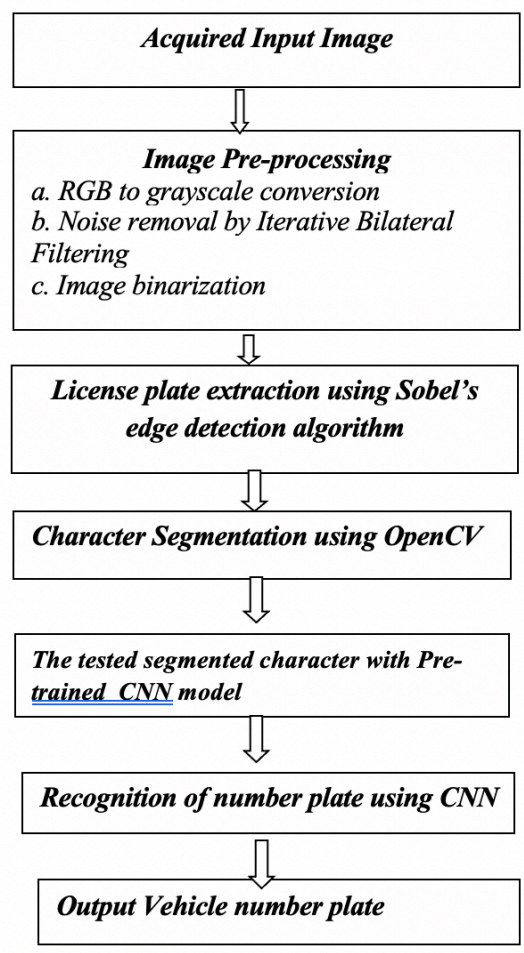

Fig. 1. Basic steps for Automatic Number System Recognition 


\subsection{Segmentation of Number Plate}

The car's number plate is taken from a high-resolution camera-the image captured in RGB format converted to a gray image. Pre-processing is applied to the image to improve the gray image's quality to a binary image. The image is first smoothed to reduce noise using Iterative Bilateral Filtering (Srisuk, S.,2014) before converting to a binary image. In our work, pre-processing is done using the Adaptive Gaussian threshold (Singh, T.R., Roy, S., Singh, O.I. et al., 2011) the neighborhood's values where the weights are a Gaussian window. In the next step, the license plate is extracted using a shape analysis method. The General License Plate has in the form of a rectangular shape.

In India, most license plates are white or yellow, and therefore can also use color analysis; the image must be in a binary image, or the edges of the image should be detected. We find and connect to the relevant rectangular corners. Finally, the areas connected to the box are linked, and all rectangular areas of interest are extracted. After the license plate has been extracted, each character must be fragmented. From the extracted image of the number plate, we will have to extract the uni-character images. Segmentation is one of the most important processes for the automatic identification of number plates; because all other steps depend on it. If the segmentation fails, number plate recognition will not be correct. A character can be improperly divided into two pieces, or two characters can be improperly merged. The following figure (Fig 2) shows the segmentation of some car's number plate segmentation after capturing the camera's picture.

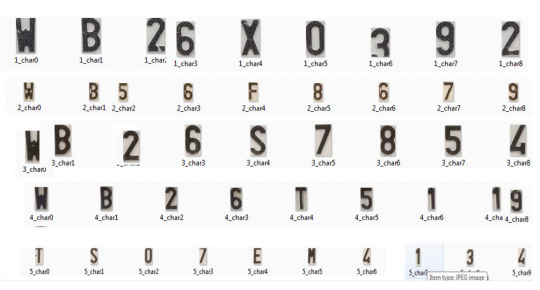

Fig. 2. Segmented character using OpenCV

\subsection{License Plate Recognition}

The final step of the automatic license plate reader system is character recognition. The number plate recognition is performed by image characters obtained at the end of the segmentation phase. This recognition's learning model must read a segmented image and convert it into the corresponding character. In order to make the most of the data available for learning, we cut each character individually by resizing it in a square after applying the same image processing steps used before segmentation of the license plate. As a result, we obtained a set of data composed of 26 classes (A to Z), and for each class, we have 30-40 images of 28X28 pixel dimensions in PNG formats; numbers from 0 to 9 and the word in English. Initially, we trained the model using the 
segmented character obtained from the application. The learning model that will be used for this recognition must read an image and render the corresponding character. (Fig 3)

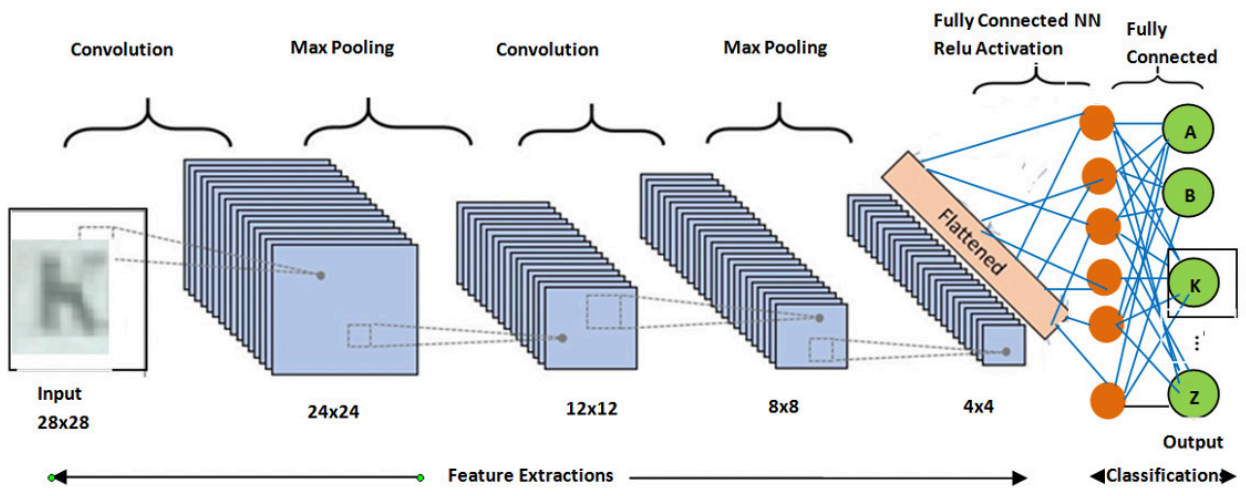

Fig. 3. Convolution Neural Network Model to recognize number plate image

In our proposed CNN algorithm has the following characteristics, it has four layers. The first layer is a convolution layer. Next two layers are hidden layer which takes weighted sums from the first. After this convolution, The ReLU activation function is applied to this layer, which forces the neurons to return positive values. The last layer uses the softmax function, representing a probability distribution of a list of potential output. We have used Adam optimizer and choose 'sparse_categorical_crossentropy' as a loss function. All proposed algorithms were done using python 3.7 with several libraries such as Open Cv, Tensorflow, and Keras. Open Cv is used, especially in the number plate localization and character segmentation stages. Tensorflow and Keras are used for the CNN algorithm, which is applied in the OCR phase and runs the simulation in the cloud environment using conda 4.5.11. We trained the model with several segmented images to obtain greater accuracy. The following figure (Fig 4) shows the Number plate recognition from a car followed by image segmentation using our proposed CNN model.

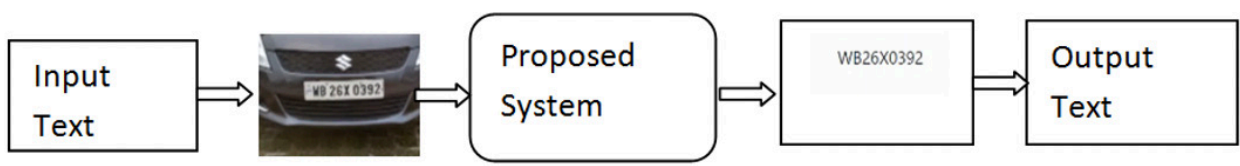

Fig 4. Automatic Number Plate Recognition using our proposed system

\section{Simulation Result and Interpretation.}

Initially, we have developed a training set database by training different segmented characters. It comprises 1,000 images of number figures where 200 images are used for testing and 800 images for training. Several tests were done by varying the Epoch value to observe the change of accuracy. The accuracy is more than $95 \%$ that presents an acceptable value compared to related works as shown in figure 5 


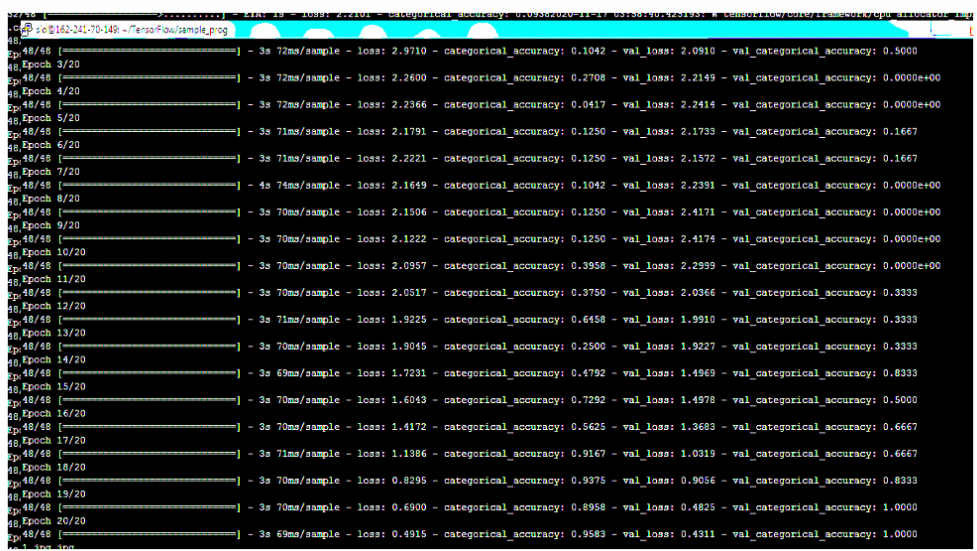

Fig 5: Simulation results show that the prediction accuracy is more than $95 \%$

At 20th Epoch we obtain the statistics as follows; Loss is $=0.4591$;Categorical Accuracy $=0.9583 ; \mathrm{Val} \_l o s s=0.4311$ and Val_categorical_accuracy $=1$

In our simulation, Val_loss is less than loss. Possible reasons are regularization (reduce the error by fitting a function appropriately) during training, but not during validation/testing. We can add regularization loss during validation/testing; loss values and curves will look more similar. Also, Training loss is measured during each epoch, while validation loss is measured after each epoch. Generally, the training loss is measured 0.5 an epoch earlier, so shift the training loss curve a half epoch to the left, losses will improve slightly.

Following simulation results (Fig 6) recognize the six number plates using our proposed simulation results.

W B 26 X 0392 '2020-08-02 11:24:34' - ProcessFrames - DEBUG - License Plate Detection completed for Vehicle 1

W B 56 F 8679 '2020-08-02 11:24:38' - ProcessFrames - DEBUG - License Plate Detection completed for Vehicle 2

W B 26 S 7854 '2020-08-02 11:24:41' - ProcessFrames - DEBUG - License Plate Detection completed for Vehicle 3

W B 76 T 5119 '2020-08-02 11:24:43' - ProcessFrames - DEBUG - License Plate Detection completed for Vehicle 4

T S 07 E M 4134 '2020-08-02 11:24:48' - ProcessFrames - DEBUG - License Plate Detection completed for Vehicle 5

W B 18 F 7716 '2020-08-02 11:24:59' - ProcessFrames - DEBUG - License Plate Detection completed for Vehicle 6

Fig 6: Simulation Results for recognizing character from number plates

\section{Limitation and Future Scope}

Our preliminary work simulated the result using a static image taken from a mobile and digital camera and further extended for real-time video streaming as a well-moving 
object. This paper has examined only fixed-length Indian number plate, which can be further generalized to the variable length plate. In our model, it can segment only a single line number plate. It cannot segment two lines number plate. Some of the Indian number plates consist of two lines; in this case, our system will not work as it cannot segment the two lines.
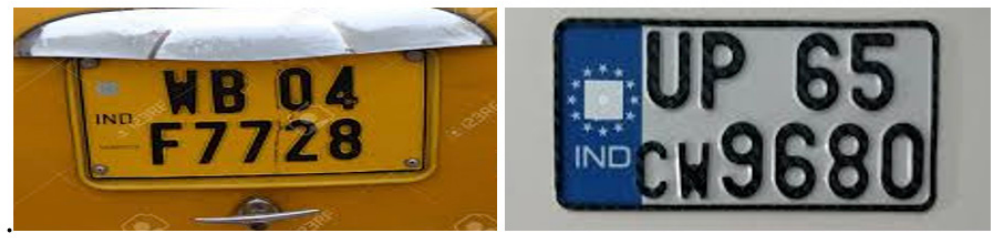

Fig. 7 Indian Number Plate consists of two line number plates.

Properly as shown in Figure 7.

Segmentation is incorrect for the images taken from various angles, as shown in Figure 8.
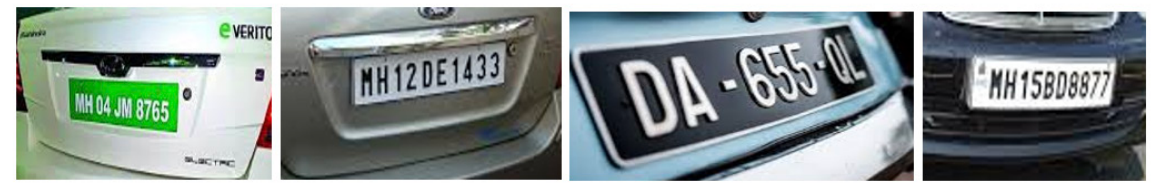

Fig 8: Capture image from various angles

Similarly, our system does not support any non-standard format. It cannot remove garbage character producing during segmentation, i.e.it does not have any noise removal capability generated during character segmentation as shown in the figure. Figure 9 shows that some garbage images are generated during segmentation, which cannot be eliminated during testing with the CNN model.

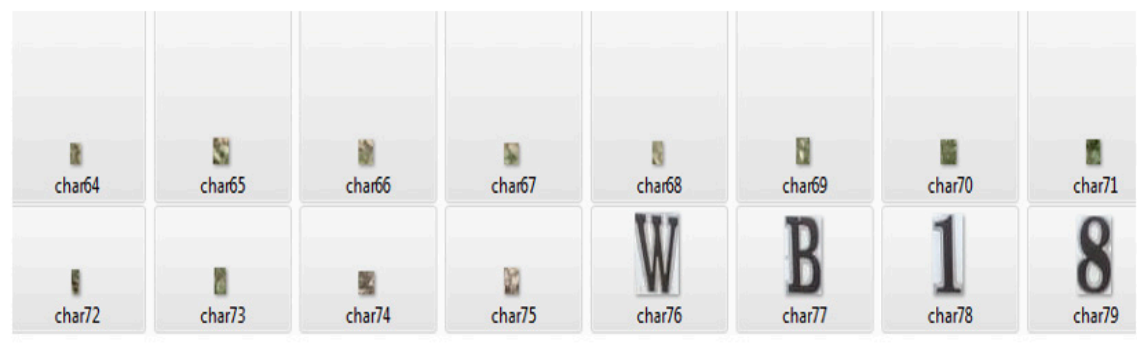

Fig 9 Garbage image generated during segmentation

We have tested only static images, but real-time video streaming is yet to be done. This paper has examined the fixed-length number plate only from the Indian 
point of view, but yet to be solved for the number plate from other countries having various lengths number plates. Support multilingual number plate, properly segment the image y taken from different angles (i.e., de-skewed the images), removal of all garbage symbols obtained during segmentation are main tasks to improve our system. This paper's future scope incorporates other features such as calculating a moving car's speed limit and detecting red light violation of a car using edge computing intel OpenVino toolkits.

\section{Conclusion}

In the NPR system, the picture of the vehicle number plate is taken with the image capturing system, and the license number is perceived with the objective that the vehicle owner's information can be easily identified. The main objective of our paper is to improve the accuracy of image number plate recognition. Even if a number plate is blurred, then our system can recognize it well. Our preliminary work simulated the result using static images taken from mobile and digital camera and further extended for real-time video streaming and capturing the images of number plate from various angles. Since our work is preliminary, main tasks such as de-skewed images, support of multilingual, and non-standard number plate have yet to be done. This paper has examined only fixed-length Indian number plate, which can be further generalized to the variable length plate. This paper's future scope incorporates other features like calculating a moving car's speed limit and detecting red light violation of a car using edge computing deep learning toolkits.

\section{References}

Abedin, M. Z., Nath, A. C., Dhar, P., Deb, K., \& Hossain, M. S. (2017, December). License plate recognition system based on contour properties and deep learning model. In 2017 IEEE Region 10 Humanitarian Technology Conference (R10-HTC) (pp. 590593). IEEE.

Abolghasemi, V., \& Ahmadyfard, A. (2009). An edge-based color-aided method for license plate detection. Image and Vision Computing, 27(8), 1134-1142.

Bulan, O., Kozitsky, V., Ramesh, P., \& Shreve, M. (2017). Segmentation-and annotation-free license plate recognition with deep localization and failure identification. IEEE Transactions on Intelligent Transportation Systems, 18(9), 2351-2363.

Conci, A., Carvalho, J., \& Rauber, T. (2009). A complete system for vehicle plate localization, segmentation and recognition in real life scene. IEEE Latin America Transactions, 7(5), 497-506.

Jia, Y., Shelhamer, E., et al. (2014, November). Caffe: Convolutional architecture for fast feature embedding. In Proceedings of the $22^{\text {nd }}$ ACM International Conference on Multimedia (pp. 675-678).

Kate, R., \& JS, D. C. (2012). Number Plate Recognition Using Segmentation. International Journal of Engineering Research \& Technology (IJERT), 1(9), 1-5. 
Kaur, E. K., \& Banga, V. K. (2013). Number plate recognition using OCR technique. International Journal of Research in Engineering and Technology, 2(09), 286290.

Krizhevsky, A., Sutskever, I., \& Hinton, G. E. (2017). Imagenet classification with deep convolutional neural networks. Communications of the ACM, 60(6), 84-90.

Li, B., Tian, B., Li, Y., \& Wen, D. (2013). Component-based license plate detection using conditional random field model. IEEE Transactions on Intelligent Transportation Systems, 14(4), 1690-1699.

Panahi, R., \& Gholampour, I. (2016). Accurate detection and recognition of dirty vehicle plate numbers for high-speed applications. IEEE Transactions on intelligent transportation systems, 18(4), 767-779.

Patel, C., Shah, D., \& Patel, A. (2013). Automatic number plate recognition system (anpr): A survey. International Journal of Computer Applications, 69(9), 21-33.

Raghunandan, K. S., Shivakumara, P., et al. (2016, April). New sharpness features for image type classification based on textual information. In $201612^{\text {th }}$ IAPR Workshop on Document Analysis Systems (DAS) (pp. 204-209). IEEE.

Roy, A., Ghoshal, D. P. (2011, March). Number Plate Recognition for use in different countries using an improved segmentation. In $20112^{\text {nd }}$ National Conference on Emerging Trends and Applications in Computer Science (pp. 1-5). IEEE.

Samra, G. A., Khalefah, F. (2013). Localization of license plate number using dynamic image processing techniques and genetic algorithms. IEEE Transactions on Evolutionary Computation, 18(2), 244-257.

Savitha, K. M., Sadashiva, V.C. (2015). An automated system for detection and recognition of vehicles number plate by using artificial neural networks, ISSN (ONLINE): 2395- 6151, 1(2), 26-33.

Selmi, Z., Halima, M. B., \& Alimi, A. M. (2017, November). Deep learning system for automatic license plate detection and recognition. In $201714^{\text {th }}$ IAPR International Conference on Document Analysis and Recognition (ICDAR) (Vol. 1, pp. 1132-1138). IEEE.

Shivakumara, P., Kumar, N. V., Guru, D. S., \& Tan, C. L. (2014, April). Separation of graphics (superimposed) and scene text in video frames. In $201411^{\text {th }}$ IAPR International Workshop on Document Analysis Systems (pp. 344-348). IEEE.

Singh, T. R., Roy, S., Singh, O. I., Sinam, T., \& Singh, K. (2012). A new local adaptive thresholding technique in binarization. arXiv preprint arXiv:1201.5227.

Srisuk, S. (2014, March). Bilateral filtering as a tool for image smoothing with edge preserving properties. In 2014 International Electrical Engineering Congress (iEECON) (pp. 1-4). IEEE.

Wang, F., Man, L., Wang, B., Xiao, Y., Pan, W., \& Lu, X. (2008). Fuzzy-based algorithm for color recognition of license plates. Pattern Recognition Letters, 29(7), 10071020.

Xu, J., Shivakumara, P., Lu, T., Phan, T. Q., \& Tan, C. L. (2014, August). Graphics and scene text classification in video. In $201422^{\text {nd }}$ International Conference on Pattern 
Recognition (pp. 4714-4719). IEEE.

Xu, J., Shivakumara, P., Lu, T., Phan, T. Q., \& Tan, C. L. (2014, August). Graphics and scene text classification in video. In $201422^{\text {nd }}$ International Conference on Pattern Recognition (pp. 4714-4719). IEEE.

Zhang, Z., \& Wang, C. (2012). The research of vehicle plate recognition technical based on BP neural network. Aasri Procedia, 1, 74-81.

Submitted: 09.07.2020

Accepted: 27.11.2020 